\title{
Recovering the space source term for the fractional-diffusion equation with Caputo-Fabrizio derivative
}

\author{
Le Nhat Huynh', Nguyen Hoang Luc ${ }^{2^{*}}$ (D), Dumitru Baleanu ${ }^{3,4,5}$ and Le Dinh Long ${ }^{2^{*}}$ (D)
}

*Correspondence:

nguyenhoangluc@tdmu.edu.vn; ledinhlong@tdmu.edu.vn

2Division of Applied Mathematics, Thu Dau Mot University, Binh

Duong Province, Vietnam

Full list of author information is

available at the end of the article

\section{Springer}

\begin{abstract}
This article is devoted to the study of the source function for the Caputo-Fabrizio time fractional diffusion equation. This new definition of the fractional derivative has no singularity. In other words, the new derivative has a smooth kernel. Here, we investigate the existence of the source term. Through an example, we show that this problem is ill-posed (in the sense of Hadamard), and the fractional Landweber method and the modified quasi-boundary value method are used to deal with this inverse problem and the regularized solution is also obtained. The convergence estimates are addressed for the regularized solution to the exact solution by using an a priori regularization parameter choice rule and an a posteriori parameter choice rule. In addition, we give a numerical example to illustrate the proposed method.
\end{abstract}

MSC: 26A33; 35B65; 35R11

Keywords: Source function; Fractional diffusion equation; Caputo-Fabrizio fractional derivative; Regularization method

\section{Introduction}

In recent times, the fractional calculus has been extensively studied. There are many definitions for the fractional derivative operator. The Riemann-Liouville fractional derivative and the Caputo fractional derivative are two of the most often used definitions of the fractional derivatives. Recently, in the case the kernel has no singularities, Caputo and Fabrizio have introduced a new definition of the fractional derivatives [1]. Fractional calculus is a subject with a long history and has gained great interest in different fields of applied science, and many authors considered this topic [2-12]. For example, Alrefai and Abdeljawad in [13] studied the analysis for fractional diffusion equations with fractional derivative with non-singular kernel.

In this work, we restore the space source term problem for a fractional diffusion equation with a Caputo-Fabrizio derivative. The fractional diffusion equation is discussed in this

(c) The Author(s) 2021. This article is licensed under a Creative Commons Attribution 4.0 International License, which permits use, sharing, adaptation, distribution and reproduction in any medium or format, as long as you give appropriate credit to the original author(s) and the source, provide a link to the Creative Commons licence, and indicate if changes were made. The images or other third party material in this article are included in the article's Creative Commons licence, unless indicated otherwise in a credit line to the material. If material is not included in the article's Creative Commons licence and your intended use is not permitted by statutory regulation or exceeds the permitted use, you will need to obtain permission directly from the copyright holder. To view a copy of this licence, visit http://creativecommons.org/licenses/by/4.0/. 
paper as follows:

$$
\begin{cases}{ }_{0}^{C F} \mathbb{D}_{t}^{\alpha} u+\mathscr{B} u=\mathcal{F}(\mathrm{x}, t), & \text { in } \Omega \times(0, T), \\ u(\mathrm{x}, t)=0, & \text { on } \partial \Omega \times(0, T), \\ u(\mathrm{x}, 0)=\mathrm{g}(\mathrm{x}), & \text { in } \Omega,\end{cases}
$$

where $0<\alpha<1, T>0$ is a fixed value and $\Omega \in \mathbb{R}^{d}$ is a bounded domain with sufficient smooth boundary $\partial \Omega{ }_{0}^{C F} \mathbb{D}_{t}^{\alpha}$ is the Caputo-Fabrizio operator for fractional derivatives of order $\alpha$. From [14], we have the following formula:

$$
{ }_{0}^{C F} \mathbb{D}_{t}^{\alpha} v(t)=\frac{\mathcal{A}(\alpha)}{1-\alpha}\left(v^{(1)} * \widetilde{f}\right)(t), \quad \tilde{f}(t)=\exp \left(-\frac{\alpha}{1-\alpha} t\right), \quad t \in(0, \infty),
$$

with $*$ defines the convolution and $\mathcal{A}(\alpha)$ is a normalization function such that $\mathcal{A}(0)=$ $\mathcal{A}(1)=1$.

For the spatial-fractional and temporal-fractional derivatives, there are a lot of new definitions to be presented. In [15-18], we can see more properties and applications of this new fractional derivative. In [19], the authors proved uniqueness of the solution to an initial value problem for linear fractional differential equation with $1<\alpha \leq 2$.

The problem (1.1) is associated to anomalous diffusion phenomenon in physical motivations. In the case $0<\alpha<1$, the model (1.1) is used for the super-diffusive model in heterogeneous media. Some physical background is found. For example, as regards the physical application of fractional calculus, the authors in [20] have shown that the fractional calculus is effective to account for the damping controllers which are compared to the classical derivative. The authors in [21] considered the separation and stability of solutions to nonlinear systems involving Caputo-Fabrizio derivatives.

Our purpose in this paper is to find an inversion source problem for (1.1). Assume that the source term $\mathcal{F}(\mathrm{x}, t)$ of the problem (1.1) is a forward problem, which can be split into $\mathscr{F}(\mathrm{x}) Q(t)$ where $Q(t)$ is known in advance. Hence, we want to identify the space source term $\mathscr{F}(\mathrm{x})$ by using the value of the final time $T$ as follows:

$$
\mathrm{u}(\mathrm{x}, T)=H(\mathrm{x}), \quad \mathrm{x} \in \Omega .
$$

In fact, the measurements are noised, instead of receiving the exact data $H$, we have the approximation data $H^{\varepsilon}$ that satisfies

$$
\left\|H^{\varepsilon}-H\right\|_{L^{2}(\Omega)} \leq \varepsilon
$$

where $\varepsilon>0$ is the noise level. A small error between the given observation $H^{\varepsilon}$ and the exact data $H^{\varepsilon}$ leads to a large error in the solution in output data. This is a reason why we propose some regularization methods in order to recover stable approximations for the unknown space source function.

In this paper, we apply the fractional Landweber method and modified quasi-boundary value method to restore the unknown space source function $\mathscr{F}$. The idea of the modified quasi-boundary value method mainly comes from Denche and Bessila's paper [22], where they solved the following backward heat conduction problem. For references to this 
method, the reader can see [23-25]. In [26], Klann and Ramlau introduced the fractional Landweber method for a linear ill-posed problem. We give an a posteriori choice of the regularization parameter based on an a priori bound of the exact solution that cannot be known exactly in practice. Here, the regularization parameter by the a priori rule is easier than an a posteriori rule. For references to this method, the reader can see [27]. According to this work, we can compare which method is the more optimal for the space source term problem for the fractional diffusion equation with the Caputo-Fabrizio derivative.

The paper is organized as follows. In Sect. 2, we recall some preliminary results. The exact solution, the ill-posedness of the inverse problem and the conditional stability are also discussed in Sect. 2. In Sects. 3 and 4, we present a modified quasi-boundary value method and the fractional Landweber regularization method. The convergence estimate under an a priori assumption for the exact solution and the a posteriori regularization parameter choice rule are considered in here. In Sect. 5, we show a numerical example to illustrate the proposed method.

\section{The solution and some notations}

\subsection{Some basic results}

In this section, we recall some useful results. Let us consider the operator $\mathscr{B}$ on the domain $\mathcal{D}(-\mathscr{B}):=\mathcal{H}_{0}^{1}(\Omega) \cap \mathcal{H}^{2}(\Omega)$. We assume that $-\mathscr{B}$ has eigenvalues $\lambda_{k}$ with the corresponding eigenfunction $w_{k} \in \mathcal{D}(-\mathscr{B})$.

Note $0<\lambda_{1} \leq \lambda_{2} \leq \lambda_{3} \leq \cdots \leq \lambda_{k} \leq \cdots$ and $\lambda_{k} \rightarrow \infty$ as $k \rightarrow \infty$. The negative Laplacian operator $-\Delta$ on $\mathbb{X}$ is the most popular case of $\mathscr{B}$. We have

$$
\begin{cases}\mathscr{B} w_{k}(\mathrm{x})=-\lambda_{k} w_{k}(\mathrm{x}), & \mathrm{x} \in \Omega, \\ w_{k}(\mathrm{x})=0, & \mathrm{x} \in \partial \Omega .\end{cases}
$$

From [28], we see that $\mathrm{a}_{k} \geq C k^{\frac{2}{d}}$ for $C$ is a constant and $k \in \mathbb{N}$.

For all $p \geq 0$, let us define the Hilbert scale spaces and fractional powers of $\mathscr{B}$ as follows:

$$
\begin{aligned}
& \mathscr{B}^{p} v:= \begin{cases}\sum_{k=1}^{\infty} \mathrm{a}_{k}^{p}\left\langle v, w_{k}\right\rangle w_{k}, & \text { if } p \neq 0, \\
\sum_{k=1}^{\infty}\left\langle v, w_{k}\right\rangle w_{k}, & \text { if } p=0,\end{cases} \\
& \text { and } \quad v \in \mathcal{D}\left((-\mathscr{B})^{p}\right):=\left\{v \in L^{2}(\Omega): \sum_{k=1}^{\infty} \mathrm{a}_{k}^{2 p}\left|\left\langle v, w_{k}\right\rangle\right|^{2}<\infty\right\} .
\end{aligned}
$$

And we have the following norm in the Banach space $\mathcal{D}\left((-\mathscr{B})^{p}\right)$ :

$$
\|z\|_{\mathcal{D}\left((-\mathscr{B})^{p}\right)}:=\left(\sum_{k=1}^{\infty} \mathrm{a}_{k}^{2 p}\left|\left\langle z, w_{k}\right\rangle\right|^{2}\right)^{\frac{1}{2}}, \quad z \in \mathcal{D}\left((-\mathscr{B})^{p}\right) .
$$

It is easy to see that $\|v\|_{\mathcal{D}\left((-\mathscr{B})^{p}\right)}=\left\|(-\mathscr{B})^{p} v\right\|_{\mathcal{L}^{2}(\Omega)}$. Its domain is a Hilbert space endowed $\mathcal{D}\left((-\mathscr{B})^{-p}\right)$ with the dual inner product $\langle\cdot, \cdot\rangle_{-p, p}$ taken between $\mathcal{D}\left((-\mathscr{B})^{-p}\right)$ and $\mathcal{D}\left((-\mathscr{B})^{p}\right)$. This generates the norm $\|v\|_{\mathcal{D}\left((-\mathscr{B})^{-p}\right)}=\left(\sum_{k=1}^{\infty} \mathrm{a}_{k}^{-2 p}\left|\left\langle v, w_{k}\right\rangle_{-p, p}\right|^{2}\right)^{\frac{1}{2}}$. Now, we begin with the following two lemmas. 
Lemma $2.1([29,30])$ For $0<\mathrm{k}<1, \mathrm{q}>0$ and $\mathrm{m} \in \mathbb{N}$, we obtain

$$
(1-\mathrm{k})^{\mathrm{m}} \mathrm{k}^{\mathrm{q}} \leq \mathrm{q}^{\mathrm{q}}(\mathrm{m}+1)^{-\mathrm{q}}<\mathrm{q}^{\mathrm{q}} \mathrm{m}^{-\mathrm{q}} .
$$

Lemma 2.2 For $p, \gamma, \lambda_{1}$ be positive constant. Let $\mathrm{G}$ be a function defined by

$$
G(z)=\frac{\gamma z^{2-p}}{\gamma z^{2}+\beta}, \quad z>0
$$

in which $Z_{1}=Z_{1}(p, \beta)$ and $Z_{2}=Z_{2}\left(p, \beta, \lambda_{1}\right)$. Then we have the estimate

$$
G(z) \leq \begin{cases}Z_{1} \gamma^{\frac{p}{2}}, & \text { if } 0<p<2 \\ Z_{2} \gamma, & \text { if } p \geq 2, z \geq \lambda_{1}\end{cases}
$$

Proof See [23], Lemma 2.7 .

By taking the inner product of the main equation of (1.1) with $w_{k}(x)$, we get the following equality:

$$
{ }_{0}^{C F} \mathbb{D}_{t}^{\alpha} u_{k}(t)-\lambda_{k} u_{k}(t)=\mathcal{F}_{k}(t), \quad u_{k}(0)=\mathrm{g}_{k}=\left\langle\mathrm{g}, w_{k}\right\rangle, \quad H_{k}=\left\langle H, w_{k}\right\rangle,
$$

where $u_{k}(t)=\left\langle u(\cdot, t), w_{k}(\cdot)\right\rangle, \mathcal{F}_{k}(t)=\left\langle\mathcal{F}(\cdot, t), w_{k}(\cdot)\right\rangle=Q(\tau)\left\langle\mathscr{F}, w_{k}\right\rangle$ stand for its Fourier coefficient.

Thanks to [1], the Laplace transform of the Caputo-Fabrizio derivative has the following expression:

$$
\mathcal{L}\left[{ }_{0}^{C F} \mathbb{D}_{t}^{\alpha} u(t)\right]=\frac{h \mathcal{L}[u](h)-u(0)}{h+\alpha(1-h)}, \quad h>0 .
$$

We thus get $\frac{h \mathcal{L}\left[u_{k}\right](h)-u_{k}(0)}{h+\alpha(1-h)}=\lambda_{k} \mathcal{L}\left[u_{k}\right](h)+\mathcal{L}\left[\mathcal{F}_{k}\right](h)$. By $\lambda_{k}>0$ and $\alpha \in(0,1)$, we obtain

$$
\mathcal{L}\left[u_{k}\right](h)=\frac{1}{1+\lambda_{k}(1-\alpha)}\left[\frac{1}{h+\frac{\lambda_{k} \alpha}{1+\lambda_{k}(1-\alpha)}} \mathrm{g}_{k}+\frac{\lambda_{k} \alpha}{1+\lambda_{k}(1-\alpha)} \mathcal{L}\left[\mathcal{F}_{k}\right](h)\right] .
$$

Thanks to [31], we get

$$
\begin{aligned}
u_{k}(t)= & \frac{1}{1+\lambda_{k}(1-\alpha)} \exp \left(-\frac{\alpha \lambda_{k} t}{1+\lambda_{k}(1-\alpha)}\right) g_{k} \\
& +\frac{1}{1+\lambda_{k}(1-\alpha)} \exp \left(-\frac{\alpha \lambda_{k} t}{1+\lambda_{k}(1-\alpha)}\right) \mathcal{F}_{k}(t) .
\end{aligned}
$$

This implies that the solution of Problem (1.1) is given by the Fourier series

$$
\begin{aligned}
u(x, t)= & \sum_{k=1}^{\infty}\left[\frac{1}{1+\lambda_{k}(1-\alpha)} \exp \left(-\frac{\alpha \lambda_{k} t}{1+\lambda_{k}(1-\alpha)}\right) g_{k}\right. \\
& \left.+\frac{1}{1+\lambda_{k}(1-\alpha)} \int_{0}^{t} \exp \left(-\frac{\alpha \lambda_{k}(t-s)}{1+\lambda_{k}(1-\alpha)}\right) \mathcal{F}_{k}(s) \mathrm{d} s\right] w_{k}(\mathrm{x}) .
\end{aligned}
$$


By $t=T$ in (2.7) and we get

$$
\begin{aligned}
H_{k}= & \frac{1}{1+\lambda_{k}(1-\alpha)} \exp \left(-\frac{\alpha \lambda_{k} t}{1+\lambda_{k}(1-\alpha)}\right) g_{k} \\
& +\frac{\mathscr{F}_{k}}{1+\lambda_{k}(1-\alpha)} \int_{0}^{T} \exp \left(-\frac{\alpha \lambda_{k}(t-s)}{1+\lambda_{k}(1-\alpha)}\right) Q(s) \mathrm{d} s,
\end{aligned}
$$

where $H_{k}=\left\langle H, w_{k}\right\rangle$, with $k \in \mathbb{N}$.

Lemma 2.3 Let $Q_{1}>0$, and let $Q \in C([0, T] ; \mathbb{R})$ be a positive continuous function satisfy $\inf _{t \in[0, T]}|Q(t)|=Q_{1}$. Suppose that $\|Q\|_{\infty}=\sup _{t \in[0, T]}|Q(t)|$, then we have

$$
\begin{aligned}
K_{2, \alpha}\left(T, \lambda_{1}\right) \frac{Q_{1}}{\lambda_{k}} & \leq \frac{1}{1+\lambda_{k}(1-\alpha)} \int_{0}^{T} \exp \left(-\frac{\alpha \lambda_{k}(T-s)}{1+\lambda_{k}(1-\alpha)}\right) Q(s) \mathrm{d} s \\
& \leq K_{1, \alpha}(T) \frac{\|Q\|_{\infty}}{\lambda_{k}}
\end{aligned}
$$

for all $k \in \mathbb{N}$.

Proof First, we obtain

$$
\frac{1}{1+\lambda_{k}(1-\alpha)} \int_{0}^{T} \exp \left(-\frac{\alpha \lambda_{k}(T-s)}{1+\lambda_{k}(1-\alpha)}\right) Q(s) \mathrm{d} s \leq K_{1, \alpha}(T) \frac{\|Q\|_{\infty}}{\lambda_{k}},
$$

where we noted that $\exp \left(-\frac{\alpha \lambda_{k}(T-s)}{1+\lambda_{k}(1-\alpha)}\right) \leq 1$ for $\forall t \geq 0$, and $K_{1, \alpha}(T)=\frac{T}{1-\alpha}$. Otherwise, we also get

$$
\begin{aligned}
& \frac{1}{1+\lambda_{k}(1-\alpha)} \int_{0}^{T} \exp \left(-\frac{\alpha \lambda_{k}(T-s)}{1+\lambda_{k}(1-\alpha)}\right) Q(s) \mathrm{d} s \\
& \quad=\frac{1}{1+\lambda_{k}(1-\alpha)} \exp \left(-\frac{\alpha \lambda_{k} T}{1+\lambda_{k}(1-\alpha)}\right) \int_{0}^{T} \exp \left(\frac{\alpha \lambda_{k} s}{1+\lambda_{k}(1-\alpha)}\right) Q(s) \mathrm{d} s \\
& \geq \frac{Q_{1}}{\alpha \lambda_{l}} \exp \left(-\frac{\alpha \lambda_{k} T}{1+\lambda_{k}(1-\alpha)}\right)\left(\exp \left(\frac{\alpha \lambda_{k} T}{1+\lambda_{l}(1-\alpha)}\right)-1\right) .
\end{aligned}
$$

This implies that

$$
\begin{aligned}
& \frac{1}{1+\lambda_{k}(1-\alpha)} \int_{0}^{T} \exp \left(-\frac{\alpha \lambda_{k}(T-s)}{1+\lambda_{k}(1-\alpha)}\right) Q(s) \mathrm{d} s \\
& \geq \frac{Q_{1}}{\alpha \lambda_{k}}\left(1-\exp \left(-\frac{\alpha \lambda_{k} T}{1+\lambda_{k}(1-\alpha)}\right)\right) \\
& \geq K_{2, \alpha}\left(T, \lambda_{1}\right) \frac{Q_{1}}{\lambda_{k}}
\end{aligned}
$$

where $K_{2, \alpha}\left(T, \lambda_{1}\right)=\frac{1}{\alpha}\left(1-\exp \left(-\frac{\alpha \lambda_{1} T}{1+\lambda_{1}(1-\alpha)}\right)\right)$.

\subsection{III-posedness and stability estimates}

For any $v \in L^{2}(\Omega)$, let $R: L^{2}(\Omega) \rightarrow L^{2}(\Omega)$ be the following operator:

$$
(R v)(\mathrm{x}):=\int_{\Omega} \kappa(\mathrm{y}, \mathrm{x}) v(\mathrm{y}) d \mathrm{y}
$$




$$
=\sum_{k=1}^{\infty} \frac{1}{1+\lambda_{k}(1-\alpha)} \int_{0}^{T} \exp \left(-\frac{\alpha \lambda_{k}(T-s)}{1+\lambda_{k}(1-\alpha)}\right) Q(s) \mathrm{d} s\left\langle\nu, w_{k}(\cdot)\right\rangle w_{k}(\mathrm{x})
$$

where the kernel $\kappa(\cdot, \cdot)$ satisfies

$$
\kappa(\mathrm{y}, \mathrm{x}):=\sum_{k=1}^{\infty} \frac{1}{1+\lambda_{k}(1-\alpha)} \int_{0}^{T} \exp \left(-\frac{\alpha \lambda_{k}(T-s)}{1+\lambda_{k}(1-\alpha)}\right) Q(s) \mathrm{d} s w_{k}(\mathrm{y}) w_{k}(\mathrm{x})
$$

Since our problem is of finding $\mathscr{F}$ which can be transform into $R \mathscr{F}=P$ where $P=$ $\sum_{k=1}^{\infty} P_{k} w_{k}$, with $P_{k}:=H_{k}-\frac{1}{1+\lambda_{k}(1-\alpha)} \exp \left(-\frac{\alpha \lambda_{k} t}{1+\lambda_{k}(1-\alpha)}\right) \mathrm{g}_{k}$, we write

$$
\mathscr{F}_{k}=\frac{P_{k}}{\frac{1}{1+\lambda_{k}(1-\alpha)} \int_{0}^{T} \exp \left(-\frac{\alpha \lambda_{k}(T-s)}{1+\lambda_{k}(1-\alpha)}\right) Q(s) \mathrm{d} s} .
$$

It is easy to see that $R: L^{2}(\Omega) \rightarrow L^{2}(\Omega)$ is compact operator. It leads to the Problem (1.1) to be ill-posed. To show the ill-posedness of this problem, we propose an illustrative example. Assume that $\mathrm{g}=0$, we choose the final data $H^{m}(\mathrm{x})=\frac{w_{m}(\mathrm{x})}{\sqrt{\lambda_{m}}}$ then the corresponding source terms are

$$
\begin{aligned}
\mathscr{F}^{m} & =\sum_{k=1}^{\infty} \frac{P_{k} w_{k}(\mathrm{x})}{\frac{1}{1+\lambda_{k}(1-\alpha)} \int_{0}^{T} \exp \left(-\frac{\alpha \lambda_{k}(T-s)}{1+\lambda_{k}(1-\alpha)}\right) Q(s) \mathrm{d} s} \\
& =\frac{1}{\frac{\sqrt{\lambda_{m}}}{1+\lambda_{m}(1-\alpha)} \int_{0}^{T} \exp \left(-\frac{\alpha \lambda_{m}(T-s)}{1+\lambda_{m}(1-\alpha)}\right) Q(s) \mathrm{d} s} .
\end{aligned}
$$

Hence, we get $\mathscr{F}^{m} \geq \frac{\sqrt{\lambda_{m}}}{\|Q\|_{\infty} K_{1, \alpha}(T)}$. This implies that $\lim _{m \rightarrow \infty}\left\|\mathscr{F}^{m}(\cdot)\right\|_{L^{2}(\Omega)} \rightarrow \infty$. But $\left\|H^{m}\right\|_{L^{2}(\Omega)}=\frac{1}{\sqrt{\lambda_{m}}}$, or $\lim _{m \rightarrow \infty}\left\|H^{m}(\cdot)\right\|_{L^{2}(\Omega)} \rightarrow 0$. Therefore, the problem (1.1) satisfying (1.2) is ill-posed in the sense of Hadamard.

\section{Modified quasi-boundary value method}

In this section, we present a regularization method to solve our problem. Now, we study a quasi-boundary value method to solve problem (1.1) and give two convergence estimates under an a priori parameter choice rule, respectively. Applying the modified quasiboundary value method given by [23], we propose the following regularized solution with exact data $P$ :

$$
\mathscr{F}_{\mathrm{n}(\varepsilon)}(\mathrm{x})=\sum_{k=1}^{\infty} \frac{\left\langle P, w_{k}\right\rangle w_{k}}{\lambda_{k}[\mathrm{n}(\varepsilon)]+\frac{1}{1+\lambda_{k}(1-\alpha)} \int_{0}^{T} \exp \left(-\frac{\alpha \lambda_{k}(T-s)}{1+\lambda_{k}(1-\alpha)}\right) Q(s) \mathrm{d} s} .
$$

We have the solution with the observation data $H^{\varepsilon}$,

$$
\mathscr{F}_{\mathrm{n}(\varepsilon)}^{\varepsilon}(\mathrm{x})=\sum_{k=1}^{\infty} \frac{\left\langle P^{\varepsilon}, w_{k}\right\rangle w_{k}}{\lambda_{k}[\mathrm{n}(\varepsilon)]+\frac{1}{1+\lambda_{k}(1-\alpha)} \int_{0}^{T} \exp \left(-\frac{\alpha \lambda_{k}(T-s)}{1+\lambda_{k}(1-\alpha)}\right) Q(s) \mathrm{d} s},
$$

here $P^{\varepsilon}:=H^{\varepsilon}-\sum_{k=1}^{\infty}\left(\frac{1}{1+\lambda_{k}(1-\alpha)} \exp \left(-\frac{\alpha \lambda_{k} T}{1+\lambda_{k}(1-\alpha)}\right) g_{k}\right) w_{k}(\mathrm{x})$ and

$$
\mathscr{F}(\mathrm{x})=\sum_{k=1}^{\infty} \frac{\left\langle P, w_{k}\right\rangle w_{k}}{\frac{1}{1+\lambda_{k}(1-\alpha)} \int_{0}^{T} \exp \left(-\frac{\alpha \lambda_{k}(T-s)}{1+\lambda_{k}(1-\alpha)}\right) Q(s) d s} .
$$


Afterwards, we will give an error estimation for $\left\|\mathscr{F}(\cdot)-\mathscr{F}_{n(\varepsilon)}^{\varepsilon}(\cdot)\right\|_{L^{2}(\Omega)}$ and show the convergence rate under a suitable choice for the regularization parameter.

Theorem 3.1 Let $H \in L^{2}(\Omega)$ and $Q:[0, T] \rightarrow \mathbb{R}$ for all $t \in[0, T]$. Assume that the a priori bound condition $\|\mathscr{F}\|_{\mathcal{D}\left((-\mathscr{B})^{p}\right)} \leq \mathcal{M}$ and the assumption (1.3) holds.

If we choose the regularization parameter

$$
\mathrm{n}(\varepsilon)= \begin{cases}\left\lfloor\left(\frac{\varepsilon}{\mathcal{M}}\right)^{\frac{2}{p+1}}\right\rfloor, & 0<p<2, \\ \left\lfloor\left(\frac{\varepsilon}{\mathcal{M}}\right)^{\frac{2}{3}}\right\rfloor, & p \geq 2\end{cases}
$$

then we get

$$
\begin{aligned}
& \left\|\mathscr{F}_{\mathrm{n}(\varepsilon)}^{\varepsilon}(\cdot)-\mathscr{F}(\cdot)\right\|_{L^{2}(\Omega)} \\
& \quad \leq \begin{cases}\left(\left(2^{-1}\left[K_{2, \alpha}\left(T, \lambda_{1}\right) Q_{1}\right]^{-\frac{1}{2}}\right)+Z_{1}\right) \mathcal{M}^{\frac{1}{p+1}} \varepsilon^{\frac{p}{p+1}}, & \text { if } 0<p<2, \\
\left(\left(2^{-1}\left[K_{2, \alpha}\left(T, \lambda_{1}\right) Q_{1}\right]^{-\frac{1}{2}}\right)+Z_{2}\right) \mathcal{M}^{\frac{1}{3}} \varepsilon^{\frac{2}{3}}, & \text { if } p \geq 2 .\end{cases}
\end{aligned}
$$

Proof Using the triangle inequality, we get

$$
\left\|\mathscr{F}_{\mathrm{n}(\varepsilon)}^{\varepsilon}(\cdot)-\mathscr{F}(\cdot)\right\|_{L^{2}(\Omega)} \leq\left\|\mathscr{F}_{\mathrm{n}(\varepsilon)}^{\varepsilon}(\cdot)-\mathscr{F}_{\mathrm{n}(\varepsilon)}(\cdot)\right\|_{L^{2}(\Omega)}+\left\|\mathscr{F}_{\mathrm{n}(\varepsilon)}(\cdot)-\mathscr{F}(\cdot)\right\|_{L^{2}(\Omega)} .
$$

First, we give an estimate for the first term $\left\|\mathscr{F}_{\mathrm{n}(\varepsilon)}^{\varepsilon}(\cdot)-\mathscr{F}_{\mathrm{n}(\varepsilon)}(\cdot)\right\|_{L^{2}(\Omega)}$, then we get

$$
\begin{aligned}
& \left\|\mathscr{F}_{\mathrm{n}(\varepsilon)}^{\varepsilon}(\cdot)-\mathscr{F}_{\mathrm{n}(\varepsilon)}(\cdot)\right\|_{L^{2}(\Omega)}^{2} \\
& \quad=\sum_{k=1}^{\infty} \frac{\left|\left\langle P^{\varepsilon}-P, w_{k}\right\rangle\right|^{2}}{\left(\lambda_{k}[\mathrm{n}(\varepsilon)]+\frac{1}{1+\lambda_{k}(1-\alpha)} \int_{0}^{T} \exp \left(-\frac{\alpha \lambda_{k}(T-s)}{1+\lambda_{k}(1-\alpha)}\right) Q(s) \mathrm{d} s\right)^{2}} \\
& \quad \leq \sup _{k \geq 1}\left(\frac{1}{\lambda_{k} \mathrm{n}(\varepsilon)+K_{2, \alpha}\left(T, \lambda_{1}\right) \frac{Q_{1}}{\lambda_{k}}}\right)^{2}\left\|P^{\varepsilon}-P\right\|_{L^{2}(\Omega)}^{2} \leq \sup _{k \geq 1}\left(\mathscr{A}_{k}^{2}\right) \varepsilon^{2} .
\end{aligned}
$$

From (3.5), applying Lemma 2.3 and the inequality $(a+b) \geq 2 \sqrt{a b}, \forall a, b \geq 0$, then we obtain

$$
\mathscr{A}_{k}^{2}=\left(\frac{1}{\lambda_{k} \mathrm{n}(\varepsilon)+K_{2, \alpha}\left(T, \lambda_{1}\right) \frac{Q_{1}}{\lambda_{k}}}\right)^{2} \leq \frac{1}{4 \mathrm{n}(\varepsilon) K_{2, \alpha}\left(T, \lambda_{1}\right) Q_{1}} .
$$

Substituting (3.6) into (3.5), we have

$$
\left\|\mathscr{F}_{\mathrm{n}(\varepsilon)}^{\varepsilon}(\cdot)-\mathscr{F}_{\mathrm{n}(\varepsilon)}(\cdot)\right\|_{L^{2}(\Omega)} \leq \frac{\varepsilon}{\sqrt{\mathrm{n}(\varepsilon)}}\left(2^{-1}\left[K_{2, \alpha}\left(T, \lambda_{1}\right) Q_{1}\right]^{-\frac{1}{2}}\right) .
$$

On the other hand, we estimate the second term $\left\|\mathscr{F}_{\mathrm{n}(\varepsilon)}(\cdot)-\mathscr{F}(\cdot)\right\|_{L^{2}(\Omega)}$, for brevity, we put $\mathscr{V}_{k, \alpha}(Q, T, s)=\frac{1}{1+\lambda_{k}(1-\alpha)} \int_{0}^{T} \exp \left(-\frac{\alpha \lambda_{k}(T-s)}{1+\lambda_{k}(1-\alpha)}\right) Q(s) \mathrm{d} s$. From (3.3) and (3.1), we obtain

$$
\mathscr{F}_{\mathrm{n}(\varepsilon)}(x)-\mathscr{F}(x)=\sum_{k=1}^{\infty}\left(\frac{\lambda_{k} \mathrm{n}(\varepsilon)}{\mathscr{F}_{k, \alpha}(Q, T, s)\left(\lambda_{k} \mathrm{n}(\varepsilon)+\mathscr{V}_{k, \alpha}(Q, T, s)\right)}\right)\left\langle P, w_{k}\right\rangle w_{k} .
$$


Using the a priori bound condition $\|\mathscr{F}\|_{\mathcal{D}\left((-\mathscr{B})^{p}\right)} \leq \mathcal{M}$, from (3.8), we show that

$$
\begin{aligned}
\left\|\mathscr{F}_{\mathrm{n}(\varepsilon)}(\cdot)-\mathscr{F}(\cdot)\right\|_{L^{2}(\Omega)}^{2} & =\sum_{k=1}^{\infty}\left(\frac{\left|<P, w_{k}>\right|}{\mathscr{V}_{k, \alpha}(Q, T, s)}\right)^{2} \lambda_{k}^{2 p}\left(\frac{\lambda_{k} \mathrm{n}(\varepsilon)}{\lambda_{k} \mathrm{n}(\varepsilon)+\mathscr{V}_{k, \alpha}(Q, T, s)}\right)^{2} \lambda_{k}^{-2 p} \\
& \leq \mathcal{M} \sup _{k \geq 1}\left(\mathcal{B}_{k}\right)^{2},
\end{aligned}
$$

in which

$$
\mathcal{B}_{k}=\frac{\lambda_{k}^{1-p} \mathrm{n}(\varepsilon)}{\lambda_{k} \mathrm{n}(\varepsilon)+\mathscr{V}_{k, \alpha}(Q, T, s)} \leq \frac{n(\varepsilon) \lambda_{k}^{2-p}}{n(\varepsilon) \lambda_{k}^{2}+K_{2, \alpha}\left(T, \lambda_{1}\right) Q_{1}} .
$$

From (3.10), applying the Lemma 2.2, by replacing $\beta=K_{2, \alpha}\left(T, \lambda_{1}\right) Q_{1}$ and $\gamma=\mathrm{n}(\varepsilon)$, we can find that

$$
\sup _{k \in \mathbb{N}} \mathcal{B}_{k} \leq \begin{cases}Z_{1}[n(\varepsilon)]^{\frac{p}{2},} & \text { if } 0<p<2 \\ Z_{2}[\mathrm{n}(\varepsilon)], & \text { if } p \geq 2 .\end{cases}
$$

Combining (3.4) to (3.11), it gives

$$
\begin{aligned}
& \left\|\mathscr{F}_{\mathrm{n}(\varepsilon)}^{\varepsilon}(\cdot)-\mathscr{F}(\cdot)\right\|_{L^{2}(\Omega)} \\
& \quad \leq \frac{\varepsilon}{\sqrt{\mathrm{n}(\varepsilon)}}\left(2^{-1}\left[K_{2, \alpha}\left(T, \lambda_{1}\right) Q_{1}\right]^{-\frac{1}{2}}\right)+ \begin{cases}Z_{1}[n(\varepsilon)]^{\frac{p}{2},} & \text { if } 0<p<2, \\
Z_{2}[\mathrm{n}(\varepsilon)], & \text { if } p \geq 2 .\end{cases}
\end{aligned}
$$

By choosing the regularization parameter $\mathrm{n}(\varepsilon)$ as follows:

$$
\mathrm{n}(\varepsilon)= \begin{cases}\left(\frac{\varepsilon}{\mathcal{M}}\right)^{\frac{2}{p+1}}, & \text { if } 0<p<2 \\ \left(\frac{\varepsilon}{\mathcal{M}}\right)^{\frac{2}{3}}, & \text { if } p \geq 2\end{cases}
$$

we conclude that

$$
\begin{aligned}
& \left\|\mathscr{F}_{\mathrm{n}(\varepsilon)}^{\varepsilon}(\cdot)-\mathscr{F}(\cdot)\right\|_{L^{2}(\Omega)} \\
& \quad \leq \begin{cases}\left(\left(2^{-1}\left[K_{2, \alpha}\left(T, \lambda_{1}\right) Q_{1}\right]^{-\frac{1}{2}}\right)+Z_{1}\right) \mathcal{M}^{\frac{1}{p+1}} \varepsilon^{\frac{p}{p+1}}, & \text { if } 0<p<2, \\
\left(\left(2^{-1}\left[K_{2, \alpha}\left(T, \lambda_{1}\right) Q_{1}\right]^{-\frac{1}{2}}\right)+Z_{2}\right) \mathcal{M}^{\frac{1}{3}} \varepsilon^{\frac{2}{3}}, & \text { if } p \geq 2 .\end{cases}
\end{aligned}
$$

The proof is finished.

\section{Fractional Landweber regularization method and convergence rate}

In this section, we propose a fractional Landweber regularization method to solve the illposed problem (1.1) satisfying (1.2). The convergence rates for the regularized solution under two parameter choice rules are also considered.

From [32], $R \mathscr{F}=P$ is equivalent to

$$
\mathscr{F}=\left(I-\mathrm{c} R^{*} R\right) \mathscr{F}+\mathrm{c} R^{*} P, \quad \text { for any } \mathrm{c}>0,
$$

where $0<\mathrm{c}<\|R\|^{-2}$ and $R^{*}$ is the adjoint operator of $R$. 
Applying the fractional Landweber method given by [26], we propose the regularized solution with exact data $H$ as follows:

$$
\begin{aligned}
& \mathscr{F}_{\mathrm{n}, \chi}(\mathrm{x}) \\
& =\sum_{k=1}^{\infty} \frac{\left[1-\left(1-\mathrm{c}\left[\frac{1}{1+\lambda_{k}(1-\alpha)} \int_{0}^{T} \exp \left(-\frac{\alpha \lambda_{k}(T-s)}{1+\lambda_{k}(1-\alpha)}\right) Q(s) \mathrm{d} s\right]^{2}\right)^{\mathrm{n}}\right]^{\chi}}{\frac{1}{1+\lambda_{k}(1-\alpha)} \int_{0}^{T} \exp \left(-\frac{\alpha \lambda_{k}(T-s)}{1+\lambda_{k}(1-\alpha)}\right) Q(s) \mathrm{d} s}\left\langle P, w_{k}\right\rangle w_{k} .
\end{aligned}
$$

We have the solution with the observation data noised $H^{\varepsilon}$ as follows:

$$
\begin{aligned}
& \mathscr{F}_{\mathrm{n}, \chi}^{\varepsilon}(\mathrm{x}) \\
& =\sum_{k=1}^{\infty} \frac{\left[1-\left(1-\mathrm{c}\left[\frac{1}{1+\lambda_{k}(1-\alpha)} \int_{0}^{T} \exp \left(-\frac{\alpha \lambda_{k}(T-s)}{1+\lambda_{k}(1-\alpha)}\right) Q(s) \mathrm{d} s\right]^{2}\right)^{\mathrm{n}}\right]^{\chi}}{\frac{1}{1+\lambda_{k}(1-\alpha)} \int_{0}^{T} \exp \left(-\frac{\alpha \lambda_{k}(T-s)}{1+\lambda_{k}(1-\alpha)}\right) Q(s) \mathrm{d} s}\left\langle P^{\varepsilon}, w_{k}\right\rangle w_{k},
\end{aligned}
$$

here $P^{\varepsilon}:=H^{\varepsilon}-\sum_{k=1}^{\infty}\left(\frac{1}{1+\lambda_{k}(1-\alpha)} \exp \left(-\frac{\alpha \lambda_{k} T}{1+\lambda_{k}(1-\alpha)}\right) \mathrm{g}_{k}\right) w_{k}(\mathrm{x}), \chi \in\left(\frac{1}{2}, 1\right]$ is the fractional order, and $\mathrm{n}>0$ is the iterative step and is a regularization parameter. Here, we note that when $\chi=1$, the fractional Landweber method becomes a standard Landweber regularization.

Lemma 4.1 Let $\lambda_{k}>0, \chi \in\left(\frac{1}{2}, 1\right], \mathrm{n}>0$ and $0<\mathrm{c}\left[\frac{1}{1+\lambda_{k}(1-\alpha)} \int_{0}^{T} \exp \left(-\frac{\alpha \lambda_{k}(T-s)}{1+\lambda_{k}(1-\alpha)}\right) Q(s) \mathrm{d} s\right]^{2}<1$, we obtain

$$
\sup _{k \geq 1} \frac{\left[1-\left(1-\mathrm{c}\left[\frac{1}{1+\lambda_{k}(1-\alpha)} \int_{0}^{T} \exp \left(-\frac{\alpha \lambda_{k}(T-s)}{1+\lambda_{k}(1-\alpha)}\right) Q(s) \mathrm{d} s\right]^{2}\right)^{\mathrm{n}}\right]^{\chi}}{\frac{1}{1+\lambda_{k}(1-\alpha)} \int_{0}^{T} \exp \left(-\frac{\alpha \lambda_{k}(T-s)}{1+\lambda_{k}(1-\alpha)}\right) Q(s) \mathrm{d} s} \leq \mathrm{c}^{\frac{1}{2}} \mathrm{n}^{\frac{1}{2}} .
$$

Proof First, we define $\vartheta(z):=z^{-2}\left[1-\left(1-z^{2}\right)^{\mathrm{n}}\right]^{2 \chi}$, where

$$
z^{2}:=c\left[\frac{1}{1+\lambda_{k}(1-\alpha)} \int_{0}^{T} \exp \left(-\frac{\alpha \lambda_{k}(T-s)}{1+\lambda_{k}(1-\alpha)}\right) Q(s) \mathrm{d} s\right]^{2} .
$$

It is easy to see that the function $\vartheta(z)$ is continuous in $[0,+\infty)$ when $z \in(0,1)$ and

$$
\frac{\left[1-\left(1-\mathrm{c}\left[\frac{1}{1+\lambda_{k}(1-\alpha)} \int_{0}^{T} \exp \left(-\frac{\alpha \lambda_{k}(T-s)}{1+\lambda_{k}(1-\alpha)}\right) Q(s) \mathrm{d} s\right]^{2}\right)^{\mathrm{n}}\right]^{\chi}}{\frac{1}{1+\lambda_{k}(1-\alpha)} \int_{0}^{T} \exp \left(-\frac{\alpha \lambda_{k}(T-s)}{1+\lambda_{k}(1-\alpha)}\right) Q(s) \mathrm{d} s}=\mathrm{c}^{\frac{1}{2}} \vartheta^{2}(z) .
$$

For $\chi \in\left(\frac{1}{2}, 1\right]$ and $z \in(0,1)$, applying Lemma 3.3 of [26] we get $\vartheta(z) \leq \mathrm{n}$. That infers the inequality (4.2) is correct.

\subsection{A priori parameter choice rule and convergence estimate}

Let us choose $\mathrm{n}:=\mathrm{n}(\varepsilon)$ such that $\left\|\mathscr{F}_{\mathrm{n}, \chi}^{\varepsilon}(\cdot)-\mathscr{F}(\cdot)\right\|_{L^{2}(\Omega)} \rightarrow 0$ as $\varepsilon \rightarrow 0$. Using an a priori regularization parameter choice rule, we propose the convergent rate for the fractional Landweber regularized solution $\mathscr{F}_{\mathrm{n}, x}^{\varepsilon}$ to the exact solution $\mathscr{F}$. In order to give an error estimate, we assume that $\|\mathscr{F}\|_{\mathcal{D}\left((-\mathscr{B})^{p}\right)} \leq \mathcal{M}$. Our result holds for any $p \geq 0$, where $\mathcal{M}$ is a positive constant and $\mathscr{F}$ is recalled in (2.12).

Theorem 4.2 Let $H \in L^{2}(\Omega)$ and $Q:[0, T] \rightarrow \mathbb{R}$ for all $t \in[0, T]$. Assume that the a priori bound condition $\|\mathscr{F}\|_{\mathcal{D}\left((-\mathscr{B})^{p}\right)} \leq \mathcal{M}$ and the assumption (1.3) holds. 
If we choose the regularization parameter

$$
\mathrm{n}= \begin{cases}\left\lfloor\left(\frac{\mathcal{M}}{\varepsilon}\right)^{\frac{2}{p+1}}\right\rfloor, & 0<p<2 \\ \left\lfloor\left(\frac{\mathcal{M}}{\varepsilon}\right)^{\frac{2}{3}}\right\rfloor, & p \geq 2\end{cases}
$$

then we get

$$
\begin{aligned}
& \left\|\mathscr{F}_{\mathrm{n}, \chi}^{\varepsilon}(\cdot)-\mathscr{F}(\cdot)\right\|_{L^{2}(\Omega)} \\
& \quad \leq \begin{cases}\left(\mathrm{c}+\left(\mathrm{c}\left[K_{2, \alpha}\left(T, \lambda_{1}\right) Q_{1}\right]^{2}\right)^{-\frac{p}{2}}\left(\frac{p}{2}\right)^{\frac{p}{2}}\right) \mathcal{M}^{\frac{1}{p+1}} \varepsilon^{\frac{p}{p+1}}, & 0<p<2, \\
\left(\mathrm{c}+\mathrm{c}\left[K_{2, \alpha}\left(T, \lambda_{1}\right) Q_{1}\right]^{2}\right) \mathcal{M}^{\frac{1}{3}} \varepsilon^{\frac{2}{3}}, & p \geq 2 .\end{cases}
\end{aligned}
$$

Proof From the triangle inequality, we obtain

$$
\left\|\mathscr{F}_{\mathrm{n}, \chi}^{\varepsilon}(\cdot)-\mathscr{F}(\cdot)\right\|_{L^{2}(\Omega)} \leq\left\|\mathscr{F}_{\mathrm{n}, \chi}^{\varepsilon}(\cdot)-\mathscr{F}_{\mathrm{n}, \chi}(\cdot)\right\|_{L^{2}(\Omega)}+\left\|\mathscr{F}_{\mathrm{n}, \chi}(\cdot)-\mathscr{F}(\cdot)\right\|_{L^{2}(\Omega)} .
$$

Firstly, we give an estimate for the first term $\left\|\mathscr{F}_{\mathrm{n}, \chi}^{\varepsilon}(\cdot)-\mathscr{F}_{\mathrm{n}, \chi}(\cdot)\right\|_{L^{2}(\Omega)}$. Applying Lemma 4.1, then we get

$$
\begin{aligned}
& \left\|\mathscr{F}_{\mathrm{n}, \chi}^{\varepsilon}(\cdot)-\mathscr{F}_{\mathrm{n}, \chi}(\cdot)\right\|_{L^{2}(\Omega)} \\
& \quad=\left(\sum_{k=1}^{\infty} \frac{\left[1-\left(1-\mathrm{c}\left[\frac{1}{1+\lambda_{k}(1-\alpha)} \int_{0}^{T} \exp \left(-\frac{\alpha \lambda_{k}(T-s)}{1+\lambda_{k}(1-\alpha)}\right) Q(s) \mathrm{d} s\right]^{2}\right)^{\mathrm{n}}\right]^{2 \chi}}{\left(\frac{1}{1+\lambda_{k}(1-\alpha)} \int_{0}^{T} \exp \left(-\frac{\alpha \lambda_{k}(T-s)}{1+\lambda_{k}(1-\alpha)}\right) Q(s) \mathrm{d} s\right)^{2}}\left|\left\langle P^{\varepsilon}-P, w_{k}\right\rangle\right|\right)^{\frac{1}{2}} \\
& \quad \leq \sup _{k \geq 1} \frac{\left[1-\left(1-\mathrm{c}\left[\frac{1}{1+\lambda_{k}(1-\alpha)} \int_{0}^{T} \exp \left(-\frac{\alpha \lambda_{k}(T-s)}{1+\lambda_{k}(1-\alpha)}\right) Q(s) \mathrm{d} s\right]^{2}\right)^{\mathrm{n}}\right] \chi}{\frac{1}{1+\lambda_{k}(1-\alpha)} \int_{0}^{T} \exp \left(-\frac{\alpha \lambda_{k}(T-s)}{1+\lambda_{k}(1-\alpha)}\right) Q(s) \mathrm{d} s}\left\|P^{\varepsilon}-P\right\|_{L^{2}(\Omega)} \\
& \quad \leq \mathrm{c}^{\frac{1}{2}} \mathrm{n}^{\frac{1}{2}} \varepsilon .
\end{aligned}
$$

Next, we estimate the second term,

$$
\begin{aligned}
& \left\|\mathscr{F}_{\mathrm{n}, \chi}(\cdot)-\mathscr{F}(\cdot)\right\|_{L^{2}(\Omega)} \\
& \quad=\left\|\sum_{k=1}^{\infty} \frac{1-\left[1-\left(1-\mathrm{c}\left[\frac{1}{1+\lambda_{k}(1-\alpha)} \int_{0}^{T} \exp \left(-\frac{\alpha \lambda_{k}(T-s)}{1+\lambda_{k}(1-\alpha)}\right) Q(s) \mathrm{d} s\right]^{2}\right)^{\mathrm{n}}\right]^{\chi}}{\frac{1}{1+\lambda_{k}(1-\alpha)} \int_{0}^{T} \exp \left(-\frac{\alpha \lambda_{k}(T-s)}{1+\lambda_{k}(1-\alpha)}\right) Q(s) \mathrm{d} s}\left\langle P, w_{k}\right\rangle w_{k}\right\|_{L^{2}(\Omega)} .
\end{aligned}
$$

Because $\chi \in\left(\frac{1}{2}, 1\right]$, we thus get

$$
\begin{aligned}
\left\|\mathscr{F}_{\mathrm{n}, \chi}(\cdot)-\mathscr{F}(\cdot)\right\|_{L^{2}(\Omega)} \\
\quad \leq\left\|\sum_{k=1}^{\infty}\left(1-\mathrm{c}\left[\frac{1}{1+\lambda_{k}(1-\alpha)} \int_{0}^{T} \exp \left(-\frac{\alpha \lambda_{k}(T-s)}{1+\lambda_{k}(1-\alpha)}\right) Q(s) \mathrm{d} s\right]^{2}\right)^{\mathrm{n}}\left\langle\mathscr{F}, w_{k}\right\rangle w_{k}\right\|_{L^{2}(\Omega)} .
\end{aligned}
$$

Lemma 2.3 now shows that

$$
\left\|\mathscr{F}_{\mathrm{n}, \chi}(\cdot)-\mathscr{F}(\cdot)\right\|_{L^{2}(\Omega)} \leq\left\|\sum_{k=1}^{\infty}\left(1-\mathrm{c}\left[K_{2, \alpha}\left(T, \lambda_{1}\right) \frac{Q_{1}}{\lambda_{k}}\right]^{2}\right)^{\mathrm{n}}\left\langle\mathscr{F}, w_{k}\right\rangle w_{k}\right\|_{L^{2}(\Omega)}
$$




$$
\begin{aligned}
& \leq\left(\sum_{k=1}^{\infty}\left(1-\mathrm{c}\left[K_{2, \alpha}\left(T, \lambda_{1}\right) \frac{Q_{1}}{\lambda_{k}}\right]^{2}\right)^{2 \mathrm{n}} \lambda_{k}^{2 p} \lambda_{k}^{-2 p}\left|\left\langle\mathscr{F}, w_{k}\right\rangle\right|^{2}\right)^{\frac{1}{2}} \\
& \leq \sup _{k \geq 1}\left(\left(1-\mathrm{c}\left[K_{2, \alpha}\left(T, \lambda_{1}\right) \frac{Q_{1}}{\lambda_{k}}\right]^{2}\right)^{\mathrm{n}} \lambda_{k}^{-p}\right)\left(\sum_{k=1}^{\infty} \lambda_{k}^{2 p}\left|\left\langle\mathscr{F}, w_{k}\right\rangle\right|^{2}\right)^{\frac{1}{2}} .
\end{aligned}
$$

Hence, we see that

$$
\left\|\mathscr{F}_{\mathrm{n}, \chi}^{\varepsilon}(\cdot)-\mathscr{F}(\cdot)\right\|_{L^{2}(\Omega)} \leq \mathrm{c}^{\frac{1}{2}} \mathrm{n}^{\frac{1}{2}} \varepsilon+\left(\mathrm{c}\left[K_{2, \alpha}\left(T, \lambda_{1}\right) Q_{1}\right]^{2}\right)^{-\frac{p}{2}}\left(\frac{p}{2}\right)^{\frac{p}{2}} \mathrm{n}^{-\frac{p}{2}} \mathcal{M} .
$$

Choose the regularization parameter

$$
\mathrm{n}= \begin{cases}\left\lfloor\left(\frac{\mathcal{M}}{\varepsilon}\right)^{\frac{2}{p+1}}\right\rfloor, & 0<p<2, \\ \left\lfloor\left(\frac{\mathcal{M}}{\varepsilon}\right)^{\frac{2}{3}}\right\rfloor, & p \geq 2,\end{cases}
$$

then we get

$$
\begin{aligned}
& \left\|\mathscr{F}_{\mathrm{n}, \chi}^{\varepsilon}(\cdot)-\mathscr{F}(\cdot)\right\|_{L^{2}(\Omega)} \\
& \quad \leq \begin{cases}\left(\mathrm{c}+\left(\mathrm{c}\left[K_{2, \alpha}\left(T, \lambda_{1}\right) Q_{1}\right]^{2}\right)^{-\frac{p}{2}}\left(\frac{p}{2}\right)^{\frac{p}{2}}\right) \mathcal{M}^{\frac{1}{p+1}} \varepsilon^{\frac{p}{p+1}}, & 0<p<2, \\
\left(\mathrm{c}+\mathrm{c}\left[K_{2, \alpha}\left(T, \lambda_{1}\right) Q_{1}\right]^{2}\right) \mathcal{M}^{\frac{1}{3}} \varepsilon^{\frac{2}{3}}, & p \geq 2 .\end{cases}
\end{aligned}
$$

This ends the proof.

\subsection{A posteriori parameter choice rule and convergence estimate}

Now, based on Morozov's discrepancy principle [33], we consider the choice of the a posterior regularization. Let us choose the regularization parameter $\mathrm{n}$ such that

$$
\left\|R \mathscr{F}_{\mathrm{n}, \chi}^{\varepsilon}-P^{\varepsilon}\right\|_{L^{2}(\Omega)} \leq \mu \varepsilon \leq\left\|R \mathscr{F}_{\mathrm{n}-1, \chi}^{\varepsilon}-P^{\varepsilon}\right\|_{L^{2}(\Omega)},
$$

where $\left\|P^{\varepsilon}\right\|_{L^{2}(\Omega)} \geq \mu \varepsilon>0$. Choosing $\mu>1$ and the bound for $\mathrm{n}$ is given and depends on $\varepsilon$ and $\mathcal{M}$

Theorem 4.3 If $\mathrm{n}$ satisfies (4.6), we can get the following inequality:

$$
\mathrm{n} \leq \frac{p+1}{2 \mathrm{c} K_{2, \alpha}^{2}\left(T, \lambda_{1}\right) Q_{1}^{2}}\left(\frac{\mathcal{M} K_{1, \alpha}(T)\|Q\|_{\infty}}{(\mu-1) \varepsilon}\right)^{\frac{2}{p+1}} .
$$

Then we have the following estimations

- If $0<p<1$ then

$$
\begin{aligned}
\left\|\mathscr{F}_{\mathrm{n}, \chi}^{\varepsilon}(\cdot)-\mathscr{F}(\cdot)\right\|_{L^{2}(\Omega)} \leq & {\left[\left(\frac{p+1}{2 K_{2, \alpha}^{2}\left(T, \lambda_{1}\right) Q_{1}^{2}}\right)^{\frac{1}{2}}\left(\frac{K_{1, \alpha}(T)\|Q\|_{\infty}}{(\mu-1)}\right)^{\frac{1}{p+1}}\right.} \\
& \left.+\left(\frac{1+\mu}{Q_{1} K_{2, \alpha}\left(T, \lambda_{1}\right)}\right)^{\frac{p}{p+1}}\right] \mathcal{M}^{\frac{1}{p+1}} \varepsilon^{\frac{p}{p+1}}
\end{aligned}
$$


- If $p \geq 1$ then

$$
\begin{aligned}
& \left\|\mathscr{F}_{\mathrm{n}, \chi}^{\varepsilon}(\cdot)-\mathscr{F}(\cdot)\right\|_{L^{2}(\Omega)} \\
& \quad \leq\left[\left(\frac{(p+1) K_{1, \alpha}(T)\|Q\|_{\infty}}{2(\mu-1) K_{2, \alpha}^{2}\left(T, \lambda_{1}\right) Q_{1}^{2}} \mathcal{M} \varepsilon\right)^{\frac{1}{2}}+\left(\frac{1+\mu}{Q_{1} K_{2, \alpha}\left(T, \lambda_{1}\right)}\right)^{\frac{1}{2}}\right] \mathcal{M}^{\frac{1}{2}} \varepsilon^{\frac{1}{2}} .
\end{aligned}
$$

Proof From (4.6), we have

$$
\begin{aligned}
\mu \varepsilon \leq & \left\|\mathcal{K}_{\mathscr{F}^{\varepsilon}-1, \chi}^{\varepsilon}-P^{\varepsilon}\right\|_{L^{2}(\Omega)} \\
= & \| \sum_{k=1}^{\infty}\left(\left[1-\left(1-\mathrm{c}\left[\frac{1}{1+\lambda_{k}(1-\alpha)}\right.\right.\right.\right. \\
& \left.\left.\left.\left.\times \int_{0}^{T} \exp \left(-\frac{\alpha \lambda_{k}(T-s)}{1+\lambda_{k}(1-\alpha)}\right) Q(s) \mathrm{d} s\right]^{2}\right)^{\mathrm{n}-1}\right]^{\chi}-1\right)\left\langle P^{\varepsilon}, w_{k}\right\rangle w_{k} \|_{L^{2}(\Omega)} .
\end{aligned}
$$

By $\chi \in\left(\frac{1}{2} ; 1\right]$ and $0<\mathrm{c}\left[\frac{1}{1+\lambda_{k}(1-\alpha)} \int_{0}^{T} \exp \left(-\frac{\alpha \lambda_{k}(T-s)}{1+\lambda_{k}(1-\alpha)}\right) Q(s) \mathrm{d} s\right]^{2}<1$, we obtain

$$
\mu \varepsilon \leq\left\|\mathcal{K} \mathscr{F}_{\mathrm{n}-1, \chi}^{\varepsilon}-P^{\varepsilon}\right\|_{L^{2}(\Omega)}=\left\|P^{\varepsilon}-P\right\|_{L^{2}(\Omega)}+\mathcal{O}
$$

whereby

$$
\begin{aligned}
\mathcal{O}= & \| \sum_{k=1}^{\infty}\left(1-\mathrm{c}\left[\frac{1}{1+\lambda_{k}(1-\alpha)} \int_{0}^{T} \exp \left(-\frac{\alpha \lambda_{k}(T-s)}{1+\lambda_{k}(1-\alpha)}\right) Q(s) \mathrm{d} s\right]^{2}\right)^{\mathrm{n}-1} \\
& \times\left\langle P^{\varepsilon}, w_{k}\right\rangle w_{k} \|_{L^{2}(\Omega)} .
\end{aligned}
$$

In view of Lemma 2.3 and (2.12), we get

$$
\begin{aligned}
\mathcal{O} & \leq\left\|\sum_{k=1}^{\infty}\left(1-\mathrm{c}\left[K_{2, \alpha}\left(T, \lambda_{1}\right) \frac{Q_{1}}{\lambda_{k}}\right]^{2}\right)^{\mathrm{n}-1}\left\langle P^{\varepsilon}, w_{k}\right\rangle w_{k}\right\|_{L^{2}(\Omega)} \\
& \leq\left\|\sum_{k=1}^{\infty}\left(1-\mathrm{c}\left[K_{2, \alpha}\left(T, \lambda_{1}\right) \frac{Q_{1}}{\lambda_{k}}\right]^{2}\right)^{\mathrm{n}-1}\left\langle P^{\varepsilon}, w_{k}\right\rangle w_{k}\right\|_{L^{2}(\Omega)} \\
& \leq\left(\sum_{k=1}^{\infty}\left(1-\mathrm{c}\left[K_{2, \alpha}\left(T, \lambda_{1}\right) \frac{Q_{1}}{\lambda_{k}}\right]^{2}\right)^{2(\mathrm{n}-1)} \lambda_{k}^{2 p} \lambda_{k}^{-2 p}\left|\left\langle P^{\varepsilon}, w_{k}\right\rangle\right|^{2}\right)^{\frac{1}{2}} \\
& \leq \sup _{k \geq 1}\left(\left(1-\mathrm{c}\left[K_{2, \alpha}\left(T, \lambda_{1}\right) \frac{Q_{1}}{\lambda_{k}}\right]^{2}\right)^{\mathrm{n}-1} \lambda_{k}^{-p-1} K_{1, \alpha}(T)\|Q\|_{\infty}\right)\left(\sum_{k=1}^{\infty} \lambda_{k}^{2 p}\left|\left\langle\mathscr{F}, w_{k}\right\rangle\right|^{2}\right)^{\frac{1}{2}} .
\end{aligned}
$$

By Lemma 2.1, we conclude that

$$
\mathcal{O} \leq \sup _{k \geq 1}\left(\left(1-\mathrm{c}\left[K_{2, \alpha}\left(T, \lambda_{1}\right) \frac{Q_{1}}{\lambda_{k}}\right]^{2}\right)^{\mathrm{n}-1} \lambda_{k}^{-p-1} K_{1, \alpha}(T)\|Q\|_{\infty}\right)\left(\sum_{k=1}^{\infty} \lambda_{k}^{2 p}\left|\left\langle\mathscr{F}, w_{k}\right\rangle\right|^{2}\right)^{\frac{1}{2}}
$$




$$
\leq \frac{K_{1, \alpha}(T)\|Q\|_{\infty}}{\left(c^{\frac{1}{2}} K_{2, \alpha}\left(T, \lambda_{1}\right) Q_{1}\right)^{p+1}}\left(\frac{p+1}{2}\right)^{\frac{p+1}{2}} \frac{\mathcal{M}}{\mathrm{n}^{\frac{p+1}{2}}} .
$$

We have $(\mu-1) \varepsilon \leq \frac{K_{1, \alpha}(T)\|Q\|_{\infty}}{\left(\mathrm{c}^{\frac{1}{2}} K_{2, \alpha}\left(T, \lambda_{1}\right) Q_{1}\right)^{p+1}}\left(\frac{p+1}{2}\right)^{\frac{p+1}{2}} \frac{\mathcal{M}}{\mathrm{n}^{\frac{p+1}{2}}}$. This implies that

$$
\mathrm{n} \leq \begin{cases}\frac{p+1}{2 c K_{2, \alpha}^{2}\left(T, \lambda_{1}\right) Q_{1}^{2}}\left(\frac{\mathcal{M} K_{1, \alpha}(T)\|Q\|_{\infty}}{(\mu-1) \varepsilon}\right)^{\frac{2}{p+1}}, & 0<p<1, \\ \frac{\mathcal{M}(p+1) K_{1, \alpha}(T)\|Q\|_{\infty}}{2 \varepsilon c(\mu-1) K_{2, \alpha}^{2}\left(T, \lambda_{1}\right) Q_{1}^{2}}, & p \geq 1 .\end{cases}
$$

In view of (4.4) and (4.10), we get

$$
\begin{aligned}
& \left\|\mathscr{F}_{\mathrm{n}, \chi}^{\varepsilon}(\cdot)-\mathscr{F}_{\mathrm{n}, \chi}(\cdot)\right\|_{L^{2}(\Omega)} \\
& \quad \leq \begin{cases}\left(\frac{p+1}{2 K_{2, \alpha}^{2}\left(T, \lambda_{1}\right) Q_{1}^{2}}\right)^{\frac{1}{2}}\left(\frac{K_{1, \alpha}(T)\|Q\|_{\infty}}{(\mu-1)}\right)^{\frac{1}{p+1}} \mathcal{M}^{\frac{1}{p+1}} \varepsilon^{\frac{p}{p+1}}, & 0<p<1, \\
\left(\frac{(p+1) K_{1, \alpha}(T)\|Q\|_{\infty}}{2(\mu-1) K_{2, \alpha}^{2}\left(T, \lambda_{1}\right) Q_{1}^{2}} \mathcal{M} \varepsilon\right)^{\frac{1}{2}}, & p \geq 1 .\end{cases}
\end{aligned}
$$

From (4.5) and applying Hölder's inequality, we deduce that

$$
\begin{aligned}
& \left\|\mathscr{F}_{\mathrm{n}, \chi}(\cdot)-\mathscr{F}(\cdot)\right\|_{L^{2}(\Omega)} \\
& \quad=\mathcal{P}\left\|\sum_{k=1}^{\infty} \frac{1-\left[1-\left(1-\mathrm{c}\left[\frac{1}{1+\lambda_{k}(1-\alpha)} \int_{0}^{T} \exp \left(-\frac{\alpha \lambda_{k}(T-s)}{1+\lambda_{k}(1-\alpha)}\right) Q(s) d s\right]^{2}\right)^{\mathrm{n}}\right]^{\chi}}{\frac{1}{1+\lambda_{k}(1-\alpha)} \int_{0}^{T} \exp \left(-\frac{\alpha \lambda_{k}(T-s)}{1+\lambda_{k}(1-\alpha)}\right) Q(s) \mathrm{d} s}\left\langle P, w_{k}\right\rangle w_{k}\right\|_{L^{2}(\Omega)}^{\frac{p}{p+1}} .
\end{aligned}
$$

Here

$$
\begin{aligned}
\mathcal{P} & =\left\|\sum_{k=1}^{\infty} \frac{1-\left[1-\left(1-\mathrm{c}\left[\frac{1}{1+\lambda_{k}(1-\alpha)} \int_{0}^{T} \exp \left(-\frac{\alpha \lambda_{k}(T-s)}{1+\lambda_{k}(1-\alpha)}\right) Q(s) d s\right]^{2}\right)^{\mathrm{n}}\right]^{\chi}}{\frac{1}{1+\lambda_{k}(1-\alpha)} \int_{0}^{T} \exp \left(-\frac{\alpha \lambda_{k}(T-s)}{1+\lambda_{k}(1-\alpha)}\right) Q(s) \mathrm{d} s}\left\langle P, w_{k}\right\rangle w_{k}\right\|_{L^{2}(\Omega)}^{\frac{1}{p+1}} \\
& \leq\left\|\sum_{k=1}^{\infty}\left\langle\mathscr{F}, w_{k}\right\rangle w_{k}\right\|_{L^{2}(\Omega)}^{\frac{1}{p+1}} \leq \sup _{k \geq 1}\left(\frac{1}{\lambda_{k}}\right)^{\frac{p}{p+1}}\|\mathscr{F}\|_{\mathcal{D}\left((-\mathscr{B})^{p}\right)}^{\frac{1}{p+1}},
\end{aligned}
$$

where we note that $\chi \in\left(\frac{1}{2}, 1\right]$ and

$$
0<\mathrm{c}\left[\frac{1}{1+\lambda_{k}(1-\alpha)} \int_{0}^{T} \exp \left(-\frac{\alpha \lambda_{k}(T-s)}{1+\lambda_{k}(1-\alpha)}\right) Q(s) d s\right]^{2}<1 .
$$

Hence, we obtain

$$
\begin{aligned}
& \left\|\mathscr{F}_{\mathrm{n}, \chi}(\cdot)-\mathscr{F}(\cdot)\right\|_{L^{2}(\Omega)} \\
& \leq \sup _{k \geq 1}\left(\frac{1}{\frac{\lambda_{k}}{1+\lambda_{k}(1-\alpha)} \int_{0}^{T} \exp \left(-\frac{\alpha \lambda_{k}(T-s)}{1+\lambda_{k}(1-\alpha)}\right) Q(s) \mathrm{d} s}\right)^{\frac{p}{p+1}} \\
& \times \mathcal{M}^{\frac{1}{p+1}}\left(\left\|\sum_{k=1}^{\infty}\left\langle P-P^{\varepsilon}, w_{k}\right\rangle w_{k}\right\|_{L^{2}(\Omega)}\right.
\end{aligned}
$$




$$
\begin{aligned}
& +\| \sum_{k=1}^{\infty}\left(1-\left[1-\left(1-\mathrm{c}\left[\frac{1}{1+\lambda_{k}(1-\alpha)}\right.\right.\right.\right. \\
& \left.\left.\left.\left.\left.\times \int_{0}^{T} \exp \left(-\frac{\alpha \lambda_{k}(T-s)}{1+\lambda_{k}(1-\alpha)}\right) Q(s) d s\right]^{2}\right)^{\mathrm{n}}\right]^{\chi}\right)\left\langle P^{\varepsilon}, w_{k}\right\rangle w_{k} \|_{L^{2}(\Omega)}\right)^{\frac{p}{p+1}}
\end{aligned}
$$

In view of $(4.6),\left\|\mathscr{F}_{\mathrm{n}, \chi}(\cdot)-\mathscr{F}(\cdot)\right\|_{L^{2}(\Omega)} \leq\left(\frac{1+\mu}{Q_{1} K_{2, \alpha}\left(T, \lambda_{1}\right)}\right)^{\frac{p}{p+1}} \mathcal{M}^{\frac{1}{p+1}} \varepsilon^{\frac{p}{p+1}}$. This implies the following.

- If $0<p<1$ then

$$
\begin{aligned}
\left\|\mathscr{F}_{\mathrm{n}, \chi}^{\varepsilon}(\cdot)-\mathscr{F}(\cdot)\right\|_{L^{2}(\Omega)} \leq & {\left[\left(\frac{p+1}{2 K_{2, \alpha}^{2}\left(T, \lambda_{1}\right) Q_{1}^{2}}\right)^{\frac{1}{2}}\left(\frac{K_{1, \alpha}(T)\|Q\|_{\infty}}{(\mu-1)}\right)^{\frac{1}{p+1}}\right.} \\
& \left.+\left(\frac{1+\mu}{Q_{1} K_{2, \alpha}\left(T, \lambda_{1}\right)}\right)^{\frac{p}{p+1}}\right] \mathcal{M}^{\frac{1}{p+1}} \varepsilon^{\frac{p}{p+1}}
\end{aligned}
$$

- If $p \geq 1$ then

$$
\begin{aligned}
& \left\|\mathscr{F}_{\mathrm{n}, \chi}^{\varepsilon}(\cdot)-\mathscr{F}(\cdot)\right\|_{L^{2}(\Omega)} \\
& \quad \leq\left[\left(\frac{(p+1) K_{1, \alpha}(T)\|Q\|_{\infty}}{2(\mu-1) K_{2, \alpha}^{2}\left(T, \lambda_{1}\right) Q_{1}^{2}} \mathcal{M} \varepsilon\right)^{\frac{1}{2}}+\left(\frac{1+\mu}{Q_{1} K_{2, \alpha}\left(T, \lambda_{1}\right)}\right)^{\frac{1}{2}}\right] \mathcal{M}^{\frac{1}{2}} \varepsilon^{\frac{1}{2}} .
\end{aligned}
$$

\section{Numerical test}

In this section, we show a numerical example to illustrate the proposed method. The numerical test is constructed in the following way: Firstly, we set up some computations which support this example as follows: Let $\alpha \in(0,1), T=1$ be a fixed value and $(\mathrm{x}, t) \in(0, \pi) \times(0,1)$. Then we have the eigenvalues $\lambda_{k}=k^{2}, k=1,2, \ldots$, with corresponding eigenfunction $w_{k}(\mathrm{x})=\sqrt{\frac{2}{\pi}} \sin (k \mathrm{x})$ and the inner product $\langle\cdot, \cdot\rangle_{L^{2}(0, \pi)}$ is given by

$$
\langle a, b\rangle_{L^{2}(0, \pi)}=\int_{0}^{\pi} a b \mathrm{dx} .
$$

In Mathlab software, to compute a integral, we use the code which made by Juan Camilo Medina; see Juan Camilo Medina, Simpson's Rule Integration, MATLAB Central File Exchange. Retrieved March 19, 2020.

Secondly, we choose the exact data function $\mathrm{g}(\mathrm{x})$ and $F(\mathrm{x}, t)$ as follows:

$$
\mathrm{g}(\mathrm{x})=\sqrt{\frac{2}{\pi}} \sin (\mathrm{x}), \quad F(\mathrm{x}, t)=\sqrt{\frac{2}{\pi}} t^{2} \sin (2 \mathrm{x}) .
$$

A uniform Cartesian grid can be generated as follows (see Fig. 1 as an illustration):

$$
\begin{aligned}
& \mathrm{x}_{i}=(i-1) h_{\mathrm{x}}, \quad i=1,2, \ldots, M+1, h_{\mathrm{x}}=\frac{\pi}{M}, \\
& t_{j}=(j-1) h_{t}, \quad j=1,2, \ldots, N+1, h_{t}=\frac{1}{N} .
\end{aligned}
$$


Figure 1 A uniform Cartesian grid on $(\mathrm{x}, t) \in(0, \pi) \times(0,1)$

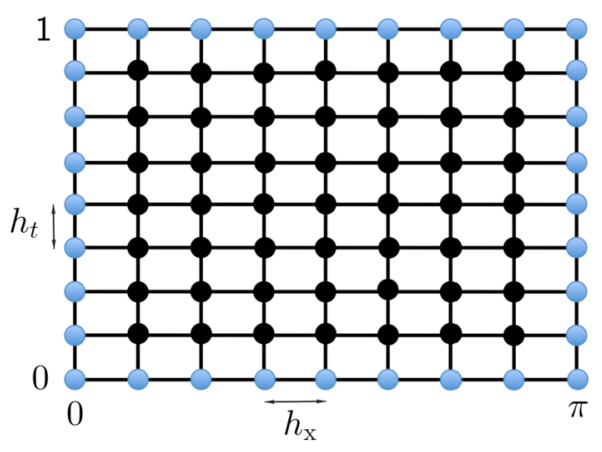

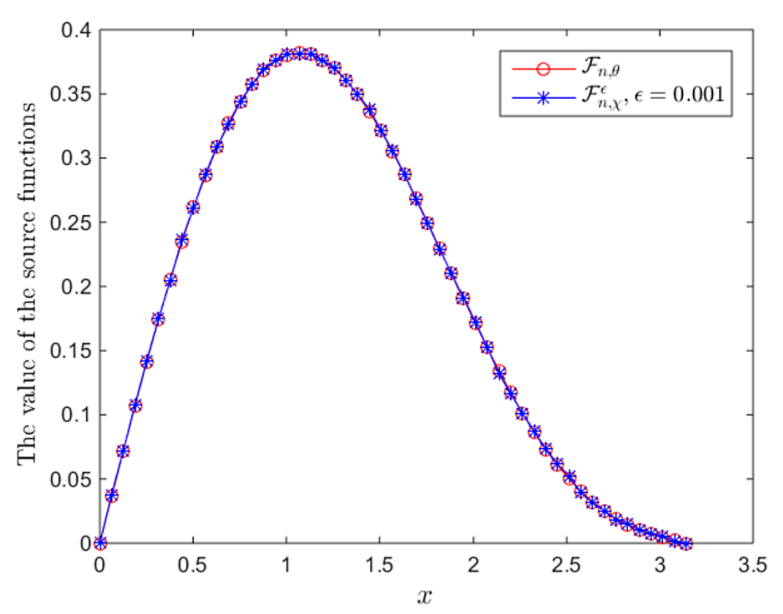

Figure 2 The solution $u(x, t)$ on $(x, t) \in(0, \pi) \times(0,1)$

Then we have the following exact solution:

$$
\begin{aligned}
& u(\mathrm{x}, t) \\
& =\sum_{k=1}^{\infty}\left[\frac{1}{1+k^{2}(1-\alpha)} \exp \left(-\frac{\alpha k^{2} t}{1+k^{2}(1-\alpha)}\right) \int_{0}^{\pi} \mathrm{g}(\mathrm{x}) \sqrt{\frac{2}{\pi}} \sin (k \mathrm{x}) \mathrm{dx}\right. \\
& \left.\quad+\frac{1}{1+k^{2}(1-\alpha)} \int_{0}^{t} \exp \left(-\frac{\alpha k^{2}(t-s)}{1+k^{2}(1-\alpha)}\right) \int_{0}^{\pi} F(\mathrm{x}, s) \sqrt{\frac{2}{\pi}} \sin (k \mathrm{x}) \mathrm{dx} \mathrm{d} s\right] \\
& \quad \times \sqrt{\frac{2}{\pi}} \sin (k \mathrm{x}) .
\end{aligned}
$$

We can see the graph of $u(\mathrm{x}, t)$ on $(\mathrm{x}, t) \in(0, \pi) \times(0,1)$ in Fig. 2 .

From (5.3), we have the value of the final time as follows:

$$
\begin{aligned}
H(\mathrm{x})= & \frac{\sqrt{2 \pi} \exp \left(\frac{\alpha}{\alpha-2}\right)}{\pi(2-\alpha)} \sin (x)+\frac{\sqrt{2 \pi} \exp \left(\frac{4 \alpha}{4 \alpha-5}\right)}{32 \pi} \\
& \times\left[\left(16 \alpha^{2}-24 \alpha+13\right) \exp \left(\frac{4}{5-4 \alpha}\right)+(5-4 \alpha)^{2}\right] \sin (2 \mathrm{x}) .
\end{aligned}
$$


Thirdly, we consider the noise model satisfies

$$
H^{\varepsilon}=H+\varepsilon \operatorname{randn}(\cdot)
$$

where the noise level $\varepsilon \longrightarrow 0^{+}$and the function $\operatorname{randn}(\cdot)$ generates arrays of random numbers whose elements are normally distributed with mean 0 , variance $\sigma^{2}=1$; see Fig. 3 .

Next, the source function with the exact data $H$ as follows:

$$
\begin{aligned}
& \mathscr{F}_{\mathrm{n}, \theta}(\mathrm{x}) \\
& =\sum_{k=1}^{\infty} \frac{\left[1-\left(1-\mathrm{c}\left[\frac{1}{1+k^{2}(1-\alpha)} \int_{0}^{1} \exp \left(-\frac{\alpha k^{2}(1-s)}{1+k^{2}(1-\alpha)}\right) Q(s) \mathrm{d} s\right]^{2}\right)^{\mathrm{n}}\right]^{\chi}}{\frac{1}{1+k^{2}(1-\alpha)} \int_{0}^{1} \exp \left(-\frac{\alpha k^{2}(1-s)}{1+k^{2}(1-\alpha)}\right) Q(s) \mathrm{d} s}\left\langle\mathscr{P}, w_{k}\right\rangle w_{k},
\end{aligned}
$$

where $\mathscr{P}_{k}:=H_{k}-\frac{1}{1+k^{2}(1-\alpha)} \exp \left(-\frac{\alpha k^{2}}{1+k^{2}(1-\alpha)}\right) g_{k}$.

According to the fractional Landweber regularization method, we have the source function with noise data $H^{\varepsilon}$ as follows:

$$
\begin{aligned}
& \mathscr{F}_{\mathrm{n}, \chi}^{\varepsilon}(\mathrm{x}) \\
& \quad=\sum_{k=1}^{\infty} \frac{\left[1-\left(1-\mathrm{c}\left[\frac{1}{1+k^{2}(1-\alpha)} \int_{0}^{1} \exp \left(-\frac{\alpha k^{2}(1-s)}{1+k^{2}(1-\alpha)}\right) Q(s) \mathrm{d} s\right]^{2}\right)^{\mathrm{n}}\right]^{\chi}}{\frac{1}{1+k^{2}(1-\alpha)} \int_{0}^{1} \exp \left(-\frac{\alpha k^{2}(1-s)}{1+k^{2}(1-\alpha)}\right) Q(s) \mathrm{d} s}\left\langle\mathscr{P}^{\varepsilon}, w_{k}\right\rangle w_{k} .
\end{aligned}
$$

Here $\mathscr{P}^{\varepsilon}:=H^{\varepsilon}-\sum_{k=1}^{\infty}\left(\frac{1}{1+k^{2}(1-\alpha)} \exp \left(-\frac{\alpha k^{2}}{1+k^{2}(1-\alpha)}\right) g_{k}\right) w_{k}(\mathrm{x}), \chi \in\left(\frac{1}{2}, 1\right]$ is the fractional order, and $\mathrm{n}>0$ is a regularization parameter.

The absolute error estimation $E_{\alpha}^{\epsilon}$ between the exact source function $\mathscr{F}_{\mathrm{n}, \theta}$ and the regularized source function $\mathscr{F}_{\mathrm{n}, \chi}^{\varepsilon}$ is as follows:

$$
E_{\alpha}^{\epsilon}=\left(\frac{1}{M+1} \sum_{i=1}^{M+1}\left|\mathscr{F}_{\mathrm{n}, \theta}\left(\mathrm{x}_{i}\right)-\mathscr{F}_{\mathrm{n}, \chi}^{\varepsilon}\left(\mathrm{x}_{i}\right)\right|^{2}\right)^{1 / 2}
$$

The results of this example are shown in Table 1. In Fig. 4, we present the graph of the exact and regularized source functions. From the error table and the figures above, we

\begin{tabular}{|c|c|c|c|c|}
\hline \multirow[t]{2}{*}{$\alpha$} & \multicolumn{4}{|c|}{$M=N=50, N(k)=10, n=5, c=1, \chi=3 / 4$} \\
\hline & $\varepsilon$ & 0.1 & 0.01 & 0.001 \\
\hline 0.1 & & 0.625292032114638 & 0.087100745983566 & $2.43525380437 \mathrm{e}-04$ \\
\hline 0.3 & & 0.421470519953221 & 0.001482709063832 & $1.27369718370 \mathrm{e}-04$ \\
\hline 0.5 & & 0.013836342900140 & 0.001320217219321 & $1.44739318526 \mathrm{e}-04$ \\
\hline 0.7 & & 0.030298174314198 & 0.002973010099701 & $3.39046203147 \mathrm{e}-04$ \\
\hline 0.9 & & $0.16883894823 e e-04$ & 0.015674145530483 & $8.98583008912 \mathrm{e}-04$ \\
\hline
\end{tabular}
can see that the smaller the $\varepsilon$, the better the computed approximation. In particular, the regularized source function will gradually approach the exact source function as $\varepsilon$ tends to zero.

Table 1 The error estimation between the exact and regularized source functions for $\alpha \in\{0.1,0.3,0.5,0.7,0.9\}$ and $\mathrm{x} \in(0, \pi)$ 


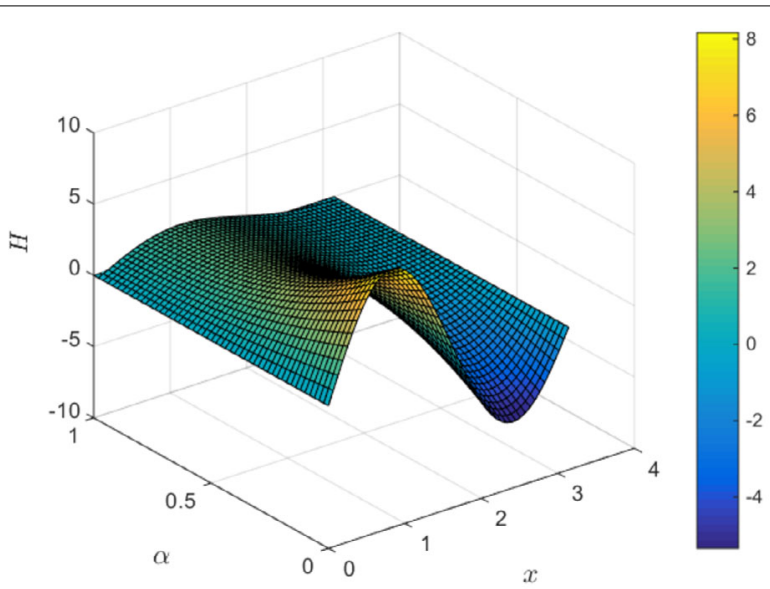

(a) $H$ for $\alpha \in(0,1)$ and $\mathrm{x} \in(0, \pi)$

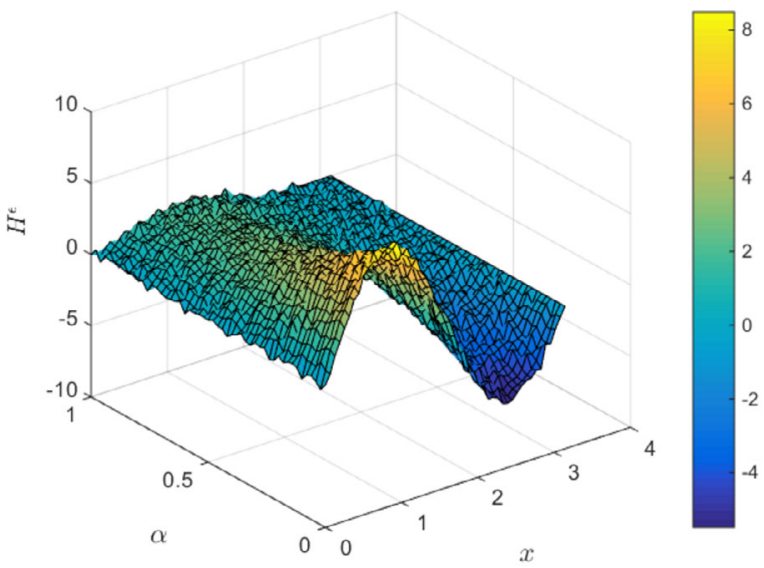

(b) $H^{\varepsilon}$ for $\alpha \in(0,1), x \in(0, \pi)$ and $\varepsilon=0.1$

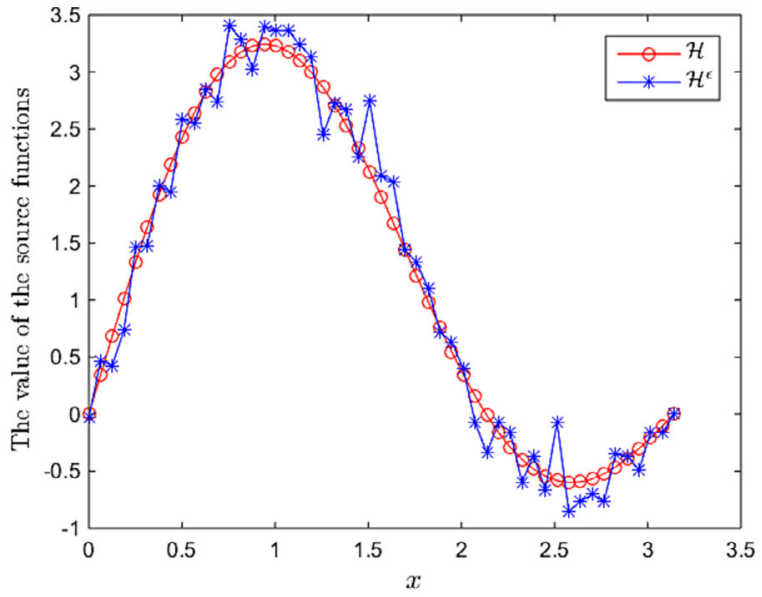

(c) $H$ and $H^{\varepsilon}$ for $\alpha=0.5, \mathrm{x} \in(0, \pi)$ and $\varepsilon=0.1$

Figure 3 The graph of functions $H$ and $H^{\varepsilon}$ 
Huynh et al. Journal of Inequalities and Applications

(2021) $2021: 28$

Page 18 of 20

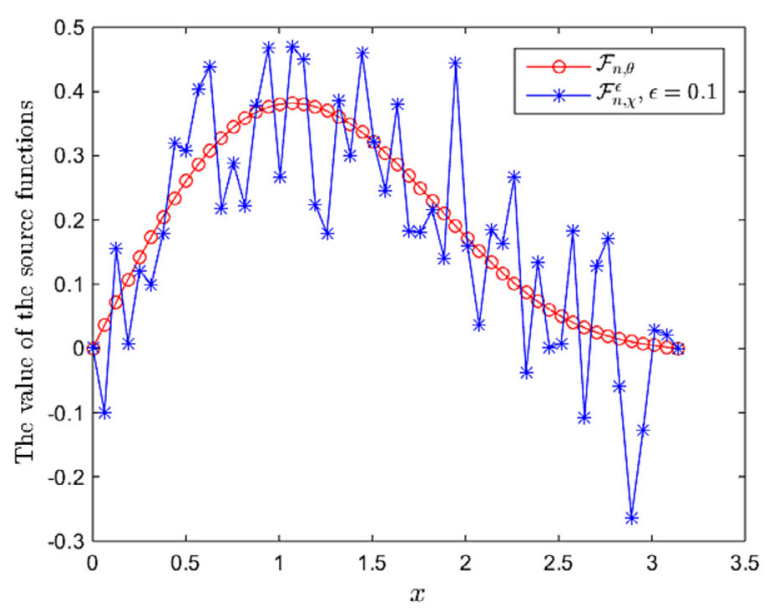

(a) $\mathscr{F}_{\mathrm{n}, \theta}$ and $\mathscr{F}_{\mathrm{n}, \mathrm{X}}^{\varepsilon}$ for $\mathrm{x} \in(0, \pi), \alpha=0.5, \varepsilon=0.1$

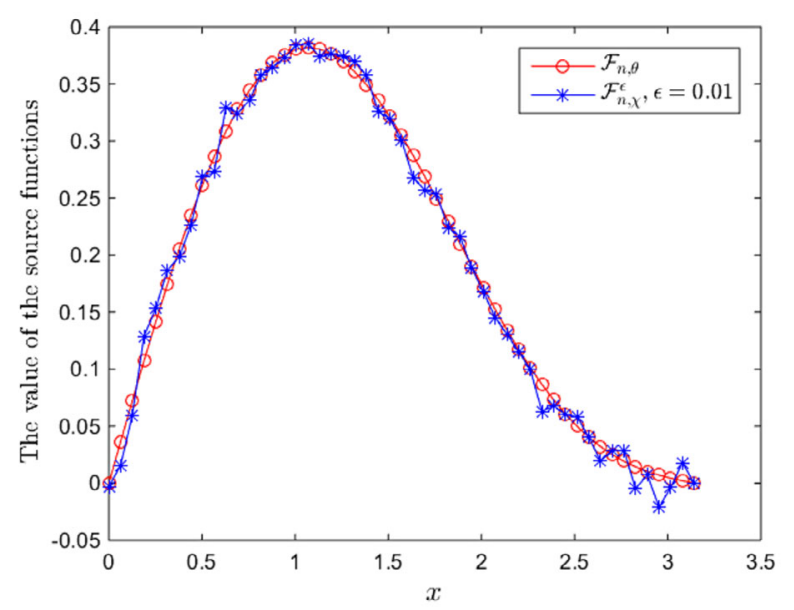

(b) $\mathscr{F}_{\mathrm{n}, \theta}$ and $\mathscr{F}_{\mathrm{n}, \chi}^{\varepsilon}$ for $\mathrm{x} \in(0, \pi), \alpha=0.5, \varepsilon=0.01$

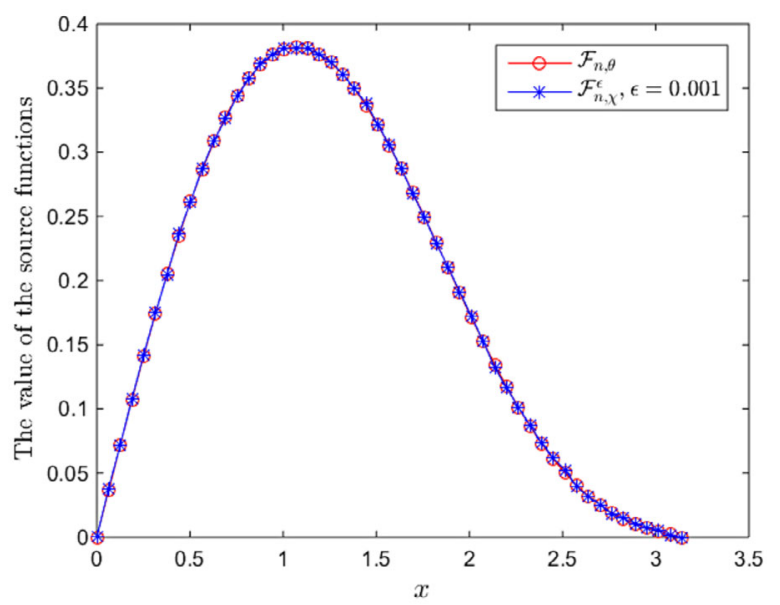

(c) $\mathscr{F}_{\mathrm{n}, \theta}$ and $\mathscr{F}_{\mathrm{n}, \mathrm{\chi}}^{\varepsilon}$ for $\mathrm{x} \in(0, \pi), \alpha=0.5, \varepsilon=0.001$

Figure 4 The exact source function $\mathscr{F}_{\mathbf{n}, \theta}$ and the regularized source function $\mathscr{F}_{\mathbf{n}, \chi}^{\varepsilon}$ for $\mathbf{x} \in(0, \pi)$ 


\section{Conclusions}

In this paper, we study the source function for the Caputo-Fabrizio time fractional diffusion equation with the new kernel. We show that the backward problem for this equation is ill-posed (in the sense of Hadamard). Then the fractional Landweber and the modified quasi-boundary value methods are used to deal with this inverse problem and the regularized solution is also obtained. We also give the convergence estimates between the regularized solution and the exact solution by using the a priori regularization parameter choice rule and the a posteriori parameter choice rule. Finally, we give a numerical example to illustrate the proposed method.

Acknowledgements
The authors would like to thank the kindness of the Editor and Reviewers in helping improve the manuscript.

Funding

Not applicable.

Availability of data and materials

Not applicable.

Competing interests

The authors declare that they have no competing interests.

Authors' contributions

The authors declare that the study was realized in collaboration with the same responsibility. All authors contributed equally to the writing of this paper. All authors read and approved the final manuscript.

\section{Author details}

${ }_{1}^{1}$ Applied Analysis Research Group, Faculty of Mathematics and Statistics, Ton Duc Thang University, Ho Chi Minh City, Vietnam. ${ }^{2}$ Division of Applied Mathematics, Thu Dau Mot University, Binh Duong Province, Vietnam. ${ }^{3}$ Department of Mathematics, Cankaya University, Ankara, Turkey. ${ }^{4}$ Department of Medical Research, China Medical University Hospital, China Medical University, Taichung, Taiwan. ${ }^{5}$ Institute of Space Sciences, Magurele-Bucharest, Romania.

\section{Publisher's Note}

Springer Nature remains neutral with regard to jurisdictional claims in published maps and institutional affiliations.

Received: 3 August 2020 Accepted: 19 January 2021 Published online: 30 January 2021

\section{References}

1. Caputo, M., Fabrizio, M.: A new definition of the fractional derivative without singular kernel. Prog. Fract. Differ. Appl. 1(2), 1-13(2015)

2. Tuan, N.H., Huynh, L.N., Baleanu, D., Can, N.H.: On a terminal value problem for a generalization of the fractional diffusion equation with hyper-Bessel operator. Math. Methods Appl. Sci. 43(6), 2858-2882 (2020)

3. Tuan, N.H., Baleanu, D., Thach, T.N., O'Regan, D., Can, N.H.: Final value problem for nonlinear time fractional reaction-diffusion equation with discrete data. J. Comput. Appl. Math. 376, 112883 (2020)

4. Tuan, N.H., Zhou, Y., Can, N.H.: Identifying inverse source for fractional diffusion equation with Riemann-Liouville derivative. Comput. Appl. Math. 39(2) 75, 1-16 (2020)

5. Atangana, A., Gómez-Aguilar, J.F.: Numerical approximation of Riemann-Liouville definition of fractional derivative: from Riemann-Liouville to Atangana-Baleanu. Numer. Methods Partial Differ. Equ. 34(5), 1502-1523 (2018) https://doi.org/10.1002/num.22195

6. Saad, K.M., Khader, M.M., Gómez-Aguilar, J.F., Baleanu, D.: Numerical solutions of the fractional Fisher's type equations with Atangana-Baleanu fractional derivative by using spectral collocation methods. Chaos, Interdiscip. J. Nonlinear Sci. 29(2), 1-13 (2019)

7. Kumar, S., Gómez Aguilar, J.F., Pandey, P.: Numerical solutions for the reaction-diffusion, diffusion - wave, and Cattaneo equations using a new operational matrix for the Caputo-Fabrizio derivative. Math. Methods Appl. Sci. 43(15), 8595-8607 (2020). https://doi.org/10.1002/mma.6517

8. Atangana, A., Gómez-Aguilar, J.F.: Decolonisation of fractional calculus rules: breaking commutativity and associativity to capture more natural phenomena. Eur. Phys. J. Plus 133, 1-23 (2018)

9. Abdeljawad, T.: Fractional operators with exponential kernels and a Lyapunov type inequality. Adv. Differ. Equ. 2017, $313(2017)$

10. Abdeljawad, T., Baleanu, D.: On fractional derivatives with exponential kernel and their discrete versions. J. Rep. Math. Phys. 80(1), 11-27 (2017)

11. Thach, T.N., Tuan, N.H, Tam, P.T.M., Can, N.H.: Identification of an inverse source problem for time-fractional diffusion equation with random noise. Math. Methods Appl. Sci. 42(1), 204-218 (2019). https://doi.org/10.1002/mma.5334

12. Tuan, N.H., Au, V.V., Can, N.H.: Regularization of initial inverse problem for strongly damped wave equation. Appl. Anal. 97(1), 69-88 (2018). https://doi.org/10.1080/00036811.2017.1359560 
13. Alrefai, M., Abdeljawad, T:: Analysis for fractional diffusion equations with fractional derivative with non-singular kernel. Adv. Differ. Equ. 2017(1), 315 (2017). https://doi.org/10.1186/s13662-017-1356-2

14. Losada, J., Nieto, J.J.: Properties of a new fractional derivative without singular kernel. Prog. Fract. Differ. Appl. 1(2), 87-92 (2015)

15. Atanacković, T.M., Pilipović, S., Zorica, D.: Properties of the Caputo-Fabrizio fractional derivative and its distributional settings. Fract. Calc. Appl. Anal. 21(1), 29-44 (2018)

16. Al-Refai, M., Pal, K.: New aspects of Caputo-Fabrizio fractional derivative. Prog. Fract. Differ. Appl. 5(2), 157-166 (2019)

17. Goufo, E.F.D.: Application of the Caputo-Fabrizio fractional derivative without singular kernel to Korteweg-de Vries-Burgers equation. Math. Model. Anal. 21(2), 188-198 (2016)

18. Yépez-Martínez, H., Gómez-Aguilar, J.F: A new modified definition of Caputo-Fabrizio fractional-order derivative and their applications to the multi step homotopy analysis method (MHAM). J. Comput. Appl. Math. 346, 247-260 (2019)

19. Al-Salti, N., Karimov, E., Sadarangani, K.: On a differential equation with Caputo-Fabrizio fractional derivative of order $1<\beta \leq 2$ and application to mass-spring-damper system. Prog. Fract. Differ. Appl. 2(4), 257-263 (2017)

20. Marinangeli, L., Alijani, F., Nia, S.H.H.: Fractional-order positive position feedback compensator for active vibration control of a smart composite plate. J. Sound Vib. 412(6), 1-16 (2018)

21. Zhon, W., Wang, L. Abdeljawad, T.: Separation and stability of solutions to nonlinear systems involving Caputo-Fabrizio derivatives. Adv. Differ. Equ. 2020, 166 (2020)

22. Denche, M., Bessila, K.: A modified quasi-boundary value method for ill-posed problems. J. Math. Anal. Appl. 301(2), 419-426 (2005)

23. Wei, T., Wang, J.: A modified quasi-boundary value method for an inverse source problem for the time-fractional diffusion equation. Appl. Numer. Math. 78, 95-111 (2014)

24. Tuan, N.H., Thach, T.N., Zhou, Y.: On a backward problem for two-dimensional time fractional wave equation with discrete random data. Evol. Equ. Control Theory 9(2), 561-579 (2020)

25. Zhang, H.W.: Modified quasi-boundary value method for a Cauchy problem of semi-linear elliptic equation. Int. J. Comput. Math. 89(12), 1689-1703 (2012)

26. Klann, E., Ramlau, R.: Regularization by fractional filter methods and data smoothing. Inverse Probl. 24(2), 025018 (2008)

27. Han, Y., Xiong, X., Xue, X.: A fractional Landweber method for solving backward time-fractional diffusion problem. Comput. Math. Appl. 78(1), 81-91 (2019)

28. Courant, R., Hilbert, D.: Methods of Mathematical Physics: Partial Differential Equations. Wiley, New York (2008)

29. Louis, A.K.: Inverse und Schlecht Gestellte Probleme. Springer, Berlin (2013)

30. Vainikko, G.M., Veretennikov, A.Y.: Iteration Procedures in III-Posed Problems. Nauka, Moscow (1986) (in Russian)

31. Al-Salti, N., Karimov, E., Kerbal, S.: Boundary-value problems for fractional heat equation involving Caputo-Fabrizio derivative. New Trends Math. Sci. 4(4), 79-89 (2016)

32. Kirsch, A.: An Introduction to the Mathematical Theory of Inverse Problem, vol. 120. Springer, Berlin (2011)

33. Engl, H.W., Hanke, M., Neubauer, A.: Regularization of Inverse Problems, vol. 375. Springer, Berlin (1996)

\section{Submit your manuscript to a SpringerOpen ${ }^{\circ}$ journal and benefit from:}

- Convenient online submission

- Rigorous peer review

- Open access: articles freely available online

- High visibility within the field

- Retaining the copyright to your article

Submit your next manuscript at $\boldsymbol{~ s p r i n g e r o p e n . c o m ~}$ 